\title{
Recycling model in developing countries: an illustration for Malaysia
}

\begin{abstract}
Most of the municipalities in Malaysia are facing the problem of getting new disposal sites as most of the existing disposal sites are nearly exhausted. Thus, waste managers should be flexible to accept new ideas and technologies particularly programmes of waste reduction and recycling in their waste management plans. This paper discusses the recycling model in Malaysia particularly the current status of waste recycling and its future prospects. Cost benefit analysis was also conducted in this study on different options of recycling. Recommendations to increase recycling are also discussed in this paper.
\end{abstract}

Keyword: Recycling model; Malaysia; Waste recycling 